\title{
Supercloseness Result of Higher Order FEM/LDG Coupled Method for Solving Singularly Perturbed Problem on S-Type Mesh
}

\author{
Shenglan Xie, ${ }^{1}$ Huonian Tu, ${ }^{2}$ and Peng $\mathrm{Zhu}^{1}$ \\ ${ }^{1}$ School of Mathematics, Physics and Information, Jiaxing University, Jiaxing, Zhejiang 314001, China \\ ${ }^{2}$ School of Information and Statistics, Guangxi University of Finance and Economics, Nanning, Guangxi 530003, China \\ Correspondence should be addressed to Peng Zhu; zhupeng.hnu@gmail.com
}

Received 17 January 2014; Accepted 26 May 2014; Published 19 June 2014

Academic Editor: Grzegorz Nowak

Copyright (C) 2014 Shenglan Xie et al. This is an open access article distributed under the Creative Commons Attribution License, which permits unrestricted use, distribution, and reproduction in any medium, provided the original work is properly cited.

we present a first supercloseness analysis for higher order FEM/LDG coupled method for solving singularly perturbed convectiondiffusion problem. Based on piecewise polynomial approximations of degree $k(k \geq 1)$, a supercloseness property of $k+1 / 2$ in DG norm is established on S-type mesh. Numerical experiments complement the theoretical results.

\section{Introduction}

In this paper we are interested in the construction and validation of high-order finite element approximations to problems of type

$$
\begin{aligned}
-\varepsilon u^{\prime \prime}-b u^{\prime}+c u & =f \quad \text { in } \Omega=(0,1), \\
u(0) & =u(1)=0,
\end{aligned}
$$

where $0<\varepsilon \ll 1$ is a small positive parameter and $b, c$, and $f$ are sufficiently smooth functions with the following properties:

$$
\begin{array}{r}
b(x) \geq \beta>0, \quad c(x) \geq 0, \quad c(x)+\frac{1}{2} b^{\prime}(x) \geq c_{0}>0, \\
\forall x \in \bar{\Omega},
\end{array}
$$

for some constants $\beta$ and $c_{0}$. This assumption guarantees that (1) has a unique solution in $H^{2}(\Omega) \cap H_{0}^{1}(\Omega)$ for all $f \in L^{2}(\Omega)$ [1]. Typically the solution of (1) has an exponential boundary layer at $x=0$.

Problem (1) is a simple model problem that helps understanding the behavior of numerical methods in presence of layers in more complex problems like the Navier-Stokes equations in fluid dynamics or convection diffusion equations in chemical reaction processes.

The smallness of $\varepsilon$ causes global unphysical oscillations if standard discretization schemes on general meshes are applied. To obtain accurate results without high computational cost, problem (1) is usually solved by strong stability numerical methods on the layer-adapted mesh, such as Shishkin-mesh (S-mesh) [2] or Bakhvalov-Shishkin mesh (B$S$ mesh) [3, 4]. In [5], a bilinear Galerkin finite element method was applied to (1) using a S-mesh, and it was shown that $\left\|u-u_{\mathrm{Gal}}^{N}\right\|_{1, \varepsilon}=\mathcal{O}\left(N^{-1} \ln N\right)$, where $\|\cdot\|_{1, \varepsilon}$ is the $\varepsilon$ weighted energy norm, $u$ is the exact solution, and $u_{\text {Gal }}^{N}$ is the computed solution. On the same method using a B-S mesh [4] improved this result to $\mathcal{O}\left(N^{-1}\right)$. Roos and Linß [6] provided the so-called S-type mesh which was a class of generalized Shishkin-mesh including S-mesh and B-S mesh.

A popular stabilization technique is the discontinuous Galerkin (DG) methods which were introduced in the early 1970s for the numerical solution of first order hyperbolic problems. Simultaneously, but quite independently, they were proposed as nonstandard schemes for the approximation of elliptic and parabolic problems. The DG methods on Smeshes for solving singularly perturbed problems (SPPs) were considered in [7-15]. Xie and her collaborators [8-10] 
investigated the superconvergence and uniform superconvergence properties of the local discontinuous Galerkin (LDG) method on S-mesh for 1D and/or 2D convection-diffusion type SPP. Zhu et al. [13] proved the uniformly convergence properties of the LDG methods with higher order elements on S-mesh for general 1D convection-diffusion and reactiondiffusion type SPPs. And recently Zhu and Zhang [14, 15] analyzed the uniform convergence properties of the LDG methods with bilinear and higher order elements on S-mesh for $2 \mathrm{D}$ SPP, respectively. On the other hand, the uniformly convergence of the NIPG method with bilinear elements on S-mesh was analyzed by Zarin and Roos [11] for 2D convection-diffusion type SPP with parabolic layers. In order to reduce the degrees of freedom of NIPG method, Roos and Zarin [7] and Zarin [12] analyzed the uniformly convergence of FEM/NIPG coupled method with bilinear element on S-mesh for 2D convection-diffusion type SPP with exponentially layers or characteristic layers. LDG method has much more advantages than the others in the DG methods family [16], but it also has more degrees of freedom than the others. By this motivation, Zhu and his collaborators $[17,18]$ analyzed the uniformly convergence property of FEM/LDG coupled method with linear/bilinear element on S-mesh for $1 \mathrm{D} / 2 \mathrm{D}$ convection-diffusion type SPP with boundary layer. Recently, Zhu and his collaborator [19] analyzed the uniformly convergence property of higher order FEM/LDG coupled method on S-mesh for 1D convection-diffusion type SPP with boundary layer.

A supercloseness property is a useful tool to prove superconvergence by postprocessing. Recently, Franz [20] numerically studies the supercloseness properties for higher order finite element methods and the streamline diffusion finite element methods on 2D Bakhvalov-Shishkin meshes. By the authors' knowledge, there is a few works about uniform supercloseness result of higher order DG method for solving SPP on S-type mesh. In this paper, we are interested in uniformly convergence properties and supercloseness properties of higher order FEM/LDG coupled method for 1D SPP of convection-diffusion type on S-type mesh. The paper is organized as follows. In Section 2, we introduce the Stype mesh and the FEM/LDG coupled method. The stability and error analysis of the FEM/LDG coupled method with higher order elements on S-type mesh is given in Section 3. A numerical example is presented in Section 4. It aims to validate our theoretical result.

In the sequel with $C$ we will denote a generic positive constant independent of the perturbation parameter $\varepsilon$ and mesh size.

\section{The S-Type Mesh and the FEM/LDG Coupled Method}

2.1. The S-Type Mesh. Let $N$ be an even integer. Denote by $\lambda$ the transition parameter which indicates where the mesh changes from fine to coarse. This parameter is given by

$$
\lambda=\min \left\{\frac{1}{2}, \frac{k+1.5}{\beta} \varepsilon \ln N\right\}
$$

where our trial space, which is defined below, comprises functions that are piecewise in $\mathscr{P}^{k}$ for some integer $k \geq 1$. Notice that $\varepsilon \ll 1$; here and below we take $\lambda=((k+$ $1.5) / \beta) \varepsilon \ln N$. Moreover, we suppose that $\varepsilon \leq N^{-1}$ which is realistic for this type of problems.

Let $\mathscr{T}_{N}=\left\{I_{j}=\left(x_{j-1}, x_{j}\right): j=1, \ldots, N\right\}$ be a partition of the domain $\Omega$. Let $\mathscr{T}_{N}=\left\{I_{j}=\left(x_{j-1}, x_{j}\right): j=1, \ldots, N\right\}$ be a partition of the domain $\Omega$ and $H=2(1-\lambda) / N$. We choose

$$
x_{j}= \begin{cases}\frac{(k+1.5) \varepsilon}{\beta} \phi\left(\frac{j}{N}\right), & j=0,1, \ldots, \frac{N}{2}, \\ \lambda+\left(j-\frac{N}{2}\right) H, & j=\frac{N}{2}+1, \ldots, N,\end{cases}
$$

where $\phi$ is a monotonically increasing mesh-generating function satisfying $\phi(0)=0$ and $\phi(1 / 2)=\ln N$. Given an arbitrary function $\phi$ fulfilling these conditions, a S-type mesh is defined.

We define a mesh-characterizing function $\psi$ that is closely related to $\phi$ by

$$
\phi=-\ln \psi,
$$

which is monotonically decreasing with $\psi(0)=1$ and $\psi(1 / 2)=N^{-1}$. Table 1 gives some examples of S-type meshes introduced in [6].

Denote the length of any subinterval $I_{j}$ by $h_{j}=x_{j}-x_{j-1}$. Some properties of S-type mesh are given in the following lemma.

Lemma 1 (see [21]). Assume that the piecewise differentiable mesh-generating function $\phi$ satisfies the conditions

$$
\max _{t \in[0,1 / 2]} \phi^{\prime}(t) \leq C N \text { or equivalently } \max _{t \in[0,1 / 2]} \frac{\left|\psi^{\prime}(t)\right|}{\psi(t)} \leq C N .
$$

Let $x_{j}, j=0,1, \ldots, N / 2$ be the points for a S-type mesh. Then, the estimates,

$$
\begin{array}{r}
h_{j} \leq C \varepsilon N^{-1} \max \left|\psi^{\prime}\right| \cdot \exp \left(\frac{\beta x_{j}}{(k+1.5) \varepsilon}\right), \quad j=1, \ldots, \frac{N}{2}, \\
h_{j}^{m} \exp \left(-\frac{\beta x_{j-1}}{\varepsilon}\right) \leq C\left(\varepsilon N^{-1} \max \left|\psi^{\prime}\right|\right)^{m}, \quad j=1, \ldots, \frac{N}{2}, \\
m \in[0, k+1.5],
\end{array}
$$

hold true.

Remark 2. Taking $\sigma=k+1.5$, the proof of this lemma is similar to the statements given on page 142 of [21]. From (6) we can also get a simpler bound

$$
h_{j} \leq C \varepsilon N^{-1} \max \phi^{\prime} \leq C \varepsilon, \quad j=1, \ldots, \frac{N}{2} .
$$

Set $\mathscr{T}_{N}^{1}=\left\{I_{j}\right\}_{j=1}^{N / 2}$ and $\mathscr{T}_{N}^{2}=\left\{I_{j}\right\}_{j=N / 2+1}^{N}$. We denote by $u\left(x_{j}^{+}\right)$and $u\left(x_{j}^{-}\right)$the values of $u$ at $x_{j}$, from the right cell and the left cell of $x_{j}$, respectively. 
2.2. The Weak Formulation of the FEM/LDG Coupled Method. The S-type mesh defined in Section 2.1 is fine on $\Omega_{1}=[0, \lambda]$ and coarse on $\Omega_{2}=[\lambda, 1]$. We discretize problem (1) by using the FEM on $\Omega_{1}$ where the mesh is fine enough and strong stable LDG method is used on coarse mesh part $\Omega_{2}$. The derived method is the so-called FEM/LDG coupled method. The motivation to this coupled approach is to construct a numerical scheme with strong stability property but has less degrees of freedom than LDG method.

Let $u^{i}=\left.u\right|_{\Omega_{i}}, i=1,2$, and $q=\left(u^{2}\right)^{\prime}$ in $\Omega_{2}$. Rewrite problem (1) as the following equivalent transmission problem:

$$
\begin{aligned}
& -\varepsilon\left(u^{1}\right)^{\prime \prime}-b\left(u^{1}\right)^{\prime}+c u^{1}=f \quad \text { in } \Omega_{1}, \\
& q-\left(u^{2}\right)^{\prime}=0 \quad \text { in } \Omega_{2}, \\
& -\varepsilon q^{\prime}-b\left(u^{2}\right)^{\prime}+c u^{2}=f \quad \text { in } \Omega_{2}, \\
& u^{1}(\lambda)=u^{2}(\lambda), \\
& \left(u^{1}\right)^{\prime}(\lambda)=q(\lambda),
\end{aligned}
$$

with boundary conditions

$$
u^{1}(0)=u^{2}(1)=0
$$

Let us now denote by $\mathscr{P}^{k}(K)$ the space of polynomials of degree at most $k$ on $K$ and define the finite element space $\mathscr{V}_{N}^{1}$ and $\mathscr{V}_{N}^{2}$ as follows:

$$
\begin{aligned}
\mathscr{V}_{N}^{1}=\left\{v^{1} \in H^{1}\left(\Omega_{1}\right): v^{1}(0)=0,\left.v^{1}\right|_{K} \in \mathscr{P}^{k}(K),\right. \\
\left.\forall K \in \mathscr{T}_{N}^{1}\right\}, \\
\mathscr{V}_{N}^{2}=\left\{v^{2} \in L^{2}\left(\Omega_{2}\right):\left.v^{2}\right|_{K} \in \mathscr{P}^{k}(K), \forall K \in \mathscr{T}_{N}^{2}\right\} .
\end{aligned}
$$

The space $\mathscr{V}_{N}^{1}$ is a standard conforming finite element space, whereas the functions in $\mathscr{V}_{N}^{2}$ are completely discontinuous across interelement boundaries.

We will search for approximate solutions $\left(U_{N}^{1}, U_{N}^{2}, Q_{N}\right)$ of (10) and (11) in the finite element space $\mathscr{V}_{N}^{1} \times \mathscr{V}_{N}^{2} \times \mathscr{V}_{N}^{2}$ that satisfy (10) and (11) in a weak sense. The FEM/LDG coupled method (see more details in $[17,22])$ for problems $(10)$ and (11) is defined as follows: find $\left(U_{N}^{1}, U_{N}^{2}, Q_{N}\right) \in \mathscr{V}_{N}^{1} \times \mathscr{V}_{N}^{2} \times \mathscr{V}_{N}^{2}$ such that

$$
\begin{gathered}
\int_{\Omega_{1}}\left[\varepsilon\left(U_{N}^{1}\right)^{\prime}+b U_{N}^{1}\right]\left(v^{1}\right)^{\prime} \mathrm{d} x+\int_{\Omega_{1}}\left(c+b^{\prime}\right) U_{N}^{1} v^{1} \mathrm{~d} x \\
-\left(\varepsilon \widehat{Q}_{N}+b \widetilde{U}_{N}^{2}\right)(\lambda) v^{1}(\lambda)=\int_{\Omega_{1}} f v^{1} \mathrm{~d} x,
\end{gathered}
$$

for all test function $v^{1} \in \mathscr{V}_{N}^{1}$, and

$$
\begin{aligned}
& \int_{I_{j}} Q_{N} w \mathrm{~d} x+\int_{I_{j}} U_{N}^{2} w^{\prime} \mathrm{d} x-\widehat{U}_{N}^{2}\left(x_{j}\right) w\left(x_{j}^{-}\right) \\
& \quad+\widehat{U}_{N}^{2}\left(x_{j-1}\right) w\left(x_{j-1}^{+}\right)=0, \\
& \int_{I_{j}}\left(\varepsilon Q_{N}+b U_{N}^{2}\right)\left(v^{2}\right)^{\prime} \mathrm{d} x \\
& \quad+\int_{I_{j}}\left(c+b^{\prime}\right) U_{N}^{2} v^{2} \mathrm{~d} x-\left(\varepsilon \widehat{Q}_{N}+b \widetilde{U}_{N}^{2}\right)\left(x_{j}\right) v^{2}\left(x_{j}^{-}\right) \\
& \quad+\left(\varepsilon \widehat{Q}_{N}+b \widetilde{U}_{N}^{2}\right)\left(x_{j-1}\right) v^{2}\left(x_{j-1}^{+}\right)=\int_{I_{j}} f v^{2} \mathrm{~d} x,
\end{aligned}
$$

for all test function $\left(w, v^{2}\right) \in \mathscr{V}_{N}^{2} \times \mathscr{V}_{N}^{2}$ and for all $I_{j} \in$ $\mathscr{T}_{N}^{2}$, where $\widehat{U}_{N}^{2}, \widetilde{U}_{N}^{2}$, and $\widehat{Q}_{N}$ are the numerical fluxes, which approximate the traces of $U_{N}^{2}$ and $Q_{N}$ on the boundary of the elements of $\mathscr{T}_{N}^{2}$. To complete the specification of the method, it only remains to define the numerical fluxes.

The Numerical Fluxes. We use the following notation to describe the numerical fluxes at the interior nodes. The average and jump of the trace of smooth function $v \in L^{2}\left(\Omega_{2}\right)$ at the interior node $x_{j}$ are given by

$$
\begin{aligned}
& \left\{v\left(x_{j}\right)\right\}=\frac{v\left(x_{j}^{+}\right)+v\left(x_{j}^{-}\right)}{2}, \\
& {\left[v\left(x_{j}\right)\right]=v\left(x_{j}^{+}\right)-v\left(x_{j}^{-}\right),}
\end{aligned}
$$

respectively. We now define the numerical fluxes $\widehat{U}_{N}^{2}$ and $\widehat{Q}_{N}$ by

$$
\begin{aligned}
& \widehat{U}_{N}^{2}\left(x_{j}\right)= \begin{cases}U_{N}^{1}(\lambda), & \text { if } j=\frac{N}{2}, \\
\left\{U_{N}^{2}\left(x_{j}\right)\right\}-\gamma\left[U_{N}^{2}\left(x_{j}\right)\right], & \\
\text { if } j=\frac{N}{2}+1, \ldots, N-1, & \\
0, & \text { if } j=N,\end{cases} \\
& \widehat{Q}_{N}\left(x_{j}\right)= \begin{cases}Q_{N}\left(\lambda^{+}\right)+\alpha\left(U_{N}^{2}\left(\lambda^{+}\right)-U_{N}^{1}(\lambda)\right), & \text { if } j=\frac{N}{2}, \\
\left\{Q_{N}\left(x_{j}\right)\right\}+\gamma\left[Q_{N}\left(x_{j}\right)\right] & \\
+\alpha\left[U_{N}^{2}\left(x_{j}\right)\right], & \\
\text { if } j=\frac{N}{2}+1, \ldots, N-1, & \text { if } j=N .\end{cases}
\end{aligned}
$$

Here the scalars $\alpha=\alpha(x)$ and $\gamma=\gamma(x)$ are auxiliary parameters. Their purpose is to enhance the stability and accuracy properties of the LDG method (see $[23,24]$ ). 
The numerical flux associated with the convection is the classical upwinding flux; namely,

$$
\widetilde{U}_{N}^{2}\left(x_{j}\right)= \begin{cases}U_{N}^{2}\left(x_{j}^{+}\right), & \text {if } j=\frac{N}{2}, \ldots, N-1, \\ 0, & \text { if } j=N .\end{cases}
$$

\section{Stability and Error Analysis of the FEM/LDG Coupled Method}

This section is devoted to the existence and uniqueness of the solution of the coupled method (13)-(15) with numerical fluxes (17)-(19) and its corresponding error analysis. Firstly, we rewrite our method in the primal form by eliminating $Q_{N}$ following Arnold et al. [16]. And then we get stability of the FEM/LDG coupled method, if the stabilization parameter $\alpha$ is taken of order $\mathcal{O}(1 / H)$. Under this condition, we obtain the higher order uniform convergence of the coupled method.

Primal Formulation. Let us introduce the space

$$
\begin{aligned}
\mathscr{V}_{N}:= & \left\{v \in L^{2}(\Omega): v^{i}=\left.v\right|_{\Omega_{i}}, v^{i} \in \mathscr{V}_{N}^{i}, i=1,2\right\} \\
& \mathscr{V}(N)=\left[H^{2}(\Omega) \cap H_{0}^{1}(\Omega)\right]+\mathscr{V}_{N},
\end{aligned}
$$

where

$$
\begin{gathered}
H_{0}^{1}(\Omega)=\left\{v \in H^{1}(\Omega):\left.v\right|_{\Omega_{i}}=v^{i}, i=1,2,\right. \\
\left.v^{1}(0)=0, v^{2}\left(1^{-}\right)=0\right\} .
\end{gathered}
$$

For $v \in \mathscr{V}(N)$, we define $\mathscr{L}_{1}(v)$ as the unique element in $\mathscr{V}_{N}^{2}$ satisfying

$$
\begin{aligned}
\int_{\Omega_{2}} & \mathscr{L}_{1}(v) r \mathrm{~d} x \\
= & \sum_{j=N / 2+1}^{N-1}\left[v^{2}\left(x_{j}\right)\right]\left(\left\{r\left(x_{j}\right)\right\}+\gamma\left[r\left(x_{j}\right)\right]\right) \\
& -v^{2}\left(1^{-}\right) r\left(1^{-}\right)-\left(v^{1}(\lambda)-v^{2}\left(\lambda^{+}\right)\right) r\left(\lambda^{+}\right),
\end{aligned}
$$

for all $r \in \mathscr{V}_{N}^{2}$

As a result, from (14) we get

$$
Q_{N}=\left(U_{N}^{2}\right)^{\prime}+\mathscr{L}_{1}\left(U_{N}\right) \text { in } \mathscr{V}_{N}^{2} .
$$

Similar to the definition of $\mathscr{L}_{1}(v)$, for $v \in \mathscr{V}(N)$, we define $\mathscr{L}_{2}(v)$ as the unique element in $\mathscr{V}_{N}^{2}$ satisfying

$$
\begin{aligned}
\int_{\Omega_{2}} b \mathscr{L}_{2}(v) r \mathrm{~d} x= & \sum_{j=N / 2+1}^{N-1} b\left(x_{j}\right)\left[v^{2}\left(x_{j}\right)\right] r\left(x_{j}^{+}\right) \\
& -b(\lambda) r\left(\lambda^{+}\right)\left(v^{1}(\lambda)-v^{2}\left(\lambda^{+}\right)\right),
\end{aligned}
$$

for all $r \in \mathscr{V}_{N}^{2}$.
Following [19], using the lifting operators $\mathscr{L}_{1}(\cdot)$ and $\mathscr{L}_{2}(\cdot)$, the flux form FEM/LDG coupled method (13)-(15) with numerical fluxes (17)-(19) can be rewritten as the primal form: find $U_{N} \in \mathscr{V}_{N}$ such that

$$
\begin{aligned}
\mathscr{A}_{N}\left(U_{N}, v\right):= & \mathscr{B}_{N}\left(U_{N}, v\right)+\mathscr{C}_{N}\left(U_{N}, v\right) \\
& +\mathcal{S}_{N}\left(U_{N}, v\right)=\mathscr{F}_{N}(v), \quad \forall v \in \mathscr{V}_{N},
\end{aligned}
$$

with

$$
\begin{gathered}
\mathscr{B}_{N}(w, v)=\int_{\Omega} \varepsilon\left(w^{\prime}+\mathscr{L}_{1}(w)\right)\left(v^{\prime}+\mathscr{L}_{1}(v)\right) \mathrm{d} x, \\
\mathscr{F}_{N}(v)=\int_{\Omega} f v \mathrm{~d} x \\
\mathscr{C}_{N}(w, v)=\int_{\Omega} b w\left(v^{\prime}+\mathscr{L}_{2}(v)\right) \mathrm{d} x+\int_{\Omega}\left(c+b^{\prime}\right) w v \mathrm{~d} x, \\
\mathcal{S}_{N}(w, v)=\sum_{j=N / 2+1}^{N-1} \varepsilon \alpha\left[w^{2}\left(x_{j}\right)\right]\left[v^{2}\left(x_{j}\right)\right] \\
+\varepsilon \alpha w^{2}\left(1^{-}\right) v^{2}\left(1^{-}\right) \\
+\varepsilon \alpha\left(w^{1}(\lambda)-w^{2}\left(\lambda^{+}\right)\right) \\
\times\left(v^{1}(\lambda)-v^{2}\left(\lambda^{+}\right)\right) .
\end{gathered}
$$

Here, $\mathscr{L}_{1}(\cdot)$ and $\mathscr{L}_{2}(\cdot)$ have been defined in $L^{2}(\Omega)$ by a trivial extension.

From the following lemma, the primal formulation is consistent.

Lemma 3. Let $u$ be the exact solution of the problems (10) and (11). Then the primal form (25) has the Galerkin orthogonality property

$$
\mathscr{A}_{N}\left(u-U_{N}, v\right)=0, \quad \forall v \in \mathscr{V}_{N} .
$$

Proof. The proof is similar to Lemma 3.1 in [19].

Stability Analysis. To consider the stability of the primal form $\mathscr{A}_{N}$, we define the following norms and seminorms for $v \in$ $\mathscr{V}(N)$ :

$$
\begin{aligned}
& \mid\|v\|_{\varepsilon}^{2}=\|v\|_{0, \Omega}^{2}+\varepsilon|v|_{1, N}^{2}+\varepsilon|v|_{*}^{2}+|v|_{c}^{2}, \\
&|v|_{1, N}^{2}=\left\|\left(v^{1}\right)^{\prime}\right\|_{0, \Omega_{1}}^{2}+\sum_{j=N / 2+1}^{N}\left\|\left(v^{2}\right)^{\prime}\right\|_{0, I_{j}}^{2} \\
&|v|_{*}^{2}=\sum_{j=N / 2+1}^{N-1} \alpha\left[v^{2}\left(x_{j}\right)\right]^{2}+\alpha\left(v^{2}\left(1^{-}\right)\right)^{2}+\alpha\left(v^{1}(\lambda)-v^{2}\left(\lambda^{+}\right)\right)^{2}, \\
&|v|_{c}^{2}=\frac{1}{2} \sum_{j=N / 2+1}^{N-1} b\left(x_{j}\right)\left[v^{2}\left(x_{j}\right)\right]^{2}+\frac{1}{2} b(1)\left(v^{2}\left(1^{-}\right)\right)^{2} \\
&+\frac{1}{2} b(\lambda)\left(v^{1}(\lambda)-v^{2}\left(\lambda^{+}\right)\right)^{2},
\end{aligned}
$$

where $\|\cdot\|_{0, D}$ is the usual Sobolev norm defined on region $D$. 
According to [25] (page 422), when the coefficient $\alpha=$ $\mathcal{O}\left(H^{-1}\right)$, there exists a constant $C>0$, such that

$$
\left\|\mathscr{L}_{1}(v)\right\|_{0, \Omega} \leq C|v|_{*}, \quad v \in \mathscr{V}(N) .
$$

Lemma 4. If $\alpha=\mathcal{O}(1 / H)$, there exists a constant $C>0$, such that

$$
\mathscr{A}_{N}(v, v) \geq C|\|v\||_{\varepsilon}^{2}, \quad \forall v \in \mathscr{V}_{N}
$$

Proof. The proof is similar to Lemma 3.2 in [19].

From Lemma 4, we easily get

$$
\left\|u_{N}\right\|_{\varepsilon} \leq C\|f\|_{0, \Omega}
$$

which implies the uniqueness of the solution to (25). Further, since (25) is a linear problem over the finite-dimensional space $\mathscr{V}_{N}$, the existence of the solution follows from its uniqueness. Consequently, by (23), we get the existence and uniqueness of the solution to the problem (13)-(15) with numerical fluxes (17)-(19).

Remark 5. In fact, following [25] or [8], for any $\alpha \geq 0$, we can prove the existence and uniqueness of the solution to the problem (13)-(15) with numerical fluxes (17)-(19). In this paper, we are only interested in the special case $\alpha=\mathcal{O}\left(H^{-1}\right)$.

Error Analysis. We are now going to provide a $\varepsilon$-uniform estimate for the error $u-U_{N}$ in the norm (28). The error analysis presented in this paper relies on a priori estimate of the exact solution of (1) and a special interpolation which was firstly introduced in [26].

Lemma 6 (see [27, Lemma 1.9]). Let q be some positive integer. Consider the boundary value problem (1) with the assumption of (2). Its exact solution $u$ can be composed as $u=S+E$, where the smooth part $S$ and the layer part E satisfy

$$
\begin{array}{r}
-\varepsilon S^{\prime \prime}-b S^{\prime}+c S=f, \\
-\varepsilon E^{\prime \prime}-b E^{\prime}+c E=0, \\
\left|S^{(l)}(x)\right| \leq C, \quad\left|E^{(l)}(x)\right| \leq C \varepsilon^{-l} \exp \left(-\frac{\beta x}{\varepsilon}\right) \\
\text { for } 0 \leq l \leq q .
\end{array}
$$

Next we introduce a special interpolant in [26] that will be useful later. On each element $K=\left[x_{j-1}, x_{j}\right]$, we define $k+1$ nodal functionals $\mathcal{N}_{l}$ by

$$
\begin{array}{r}
\mathcal{N}_{0}(w)=w\left(x_{j-1}\right), \quad \mathcal{N}_{k}(w)=w\left(x_{j}\right), \\
\mathcal{N}_{l}(w)=\frac{1}{\left(x_{j}-x_{j-1}\right)^{l}} \int_{x_{j-1}}^{x_{j}}\left(x-x_{j-1}\right)^{l-1} w(x) \mathrm{d} x, \\
l=1, \ldots, k-1 .
\end{array}
$$

Now a local interpolation $\mathscr{I}_{K} w \mid \in \mathscr{P}^{k}(K)$ is defined by

$$
\mathcal{N}_{l}\left(\mathscr{I}_{K} w-w\right)=0, \quad l=0, \ldots, k
$$

which can be extended to a continuous global interpolation $\mathscr{I} w \in \mathscr{V}_{N}$ via set

$$
\left.\mathscr{I} w\right|_{K}=\mathscr{I}_{K} w, \quad \forall K \in \mathscr{T}_{N}
$$

Obviously, if $k=1$, this special interpolation is just the Lagrange linear interpolation. [26].

The following error estimate is adapted from Lemma 7 of

Lemma 7. The special interpolant has the following properties:

$$
\begin{gathered}
\left((w-\mathscr{I} w)^{\prime}, v_{N}^{\prime}\right)_{K}=0, \quad \forall v_{N} \in \mathscr{V}_{N}, \\
|w-\mathscr{I} w|_{l, K} \leq C h_{K}^{k+1-l}|w|_{k+1, K} \\
l=0,1, \ldots, k+1, \quad \forall w \in H^{k+1}(K), \\
\|w-\mathscr{I} w\|_{L^{\infty}(K)} \leq C h_{K}^{k+1}|w|_{k+1, \infty, K}, \\
\forall w \in W^{k+1, \infty}(K),
\end{gathered}
$$

where $K$ is any element of partition $\mathscr{T}_{\mathcal{N}}$ and $h_{K}$ is the length of element $K$.

Lemma 8. Assume that the piecewise differential meshgenerating function $\phi$ satisfies (6). Let the exact solution $u=S+$ $E$ of the problem (1) be decomposed into a smooth and layered part, respectively, $\mathscr{I} S$ and $\mathscr{I} E$ are the interpolants of $S$ and $E$ on a S-type mesh respectively. Then, one has $\mathscr{I} u=\mathscr{I S}+\mathscr{I} E$ and the estimates

$$
\begin{gathered}
\|u-\mathscr{I} u\|_{L^{\infty}\left(\Omega_{i}\right)} \\
\leq \begin{cases}C\left(N^{-1} \max \left|\psi^{\prime}\right|\right)^{k+1} & \text { if } i=1, \\
C N^{-(k+1)} & \text { if } i=2,\end{cases} \\
\|u-\mathscr{I} u\|_{L^{2}(\Omega)} \leq C\left(N^{-1} \max \left|\psi^{\prime}\right|\right)^{k+1}, \\
|S-\mathscr{I} S|_{1, \Omega} \leq C N^{-k}, \\
|E-\mathscr{I} E|_{1, \Omega_{1}} \leq C \varepsilon^{-1 / 2}\left(N^{-1} \max \left|\psi^{\prime}\right|\right)^{k}, \\
|E-\mathscr{I} E|_{1, \Omega_{2}} \leq C N^{-(k+1)} .
\end{gathered}
$$

Proof. Our proof is based on arguments given by Tobiska [26]. The linearity of the interpolation operator implies $\mathscr{I} u=$ $\mathscr{I}(S+E)=\mathscr{I} S+\mathscr{I} E$. have

(i) The proof of (38): by Lemma 1, Lemma 7, and (33), we

$$
\begin{aligned}
\| S- & \mathscr{I} S \|_{L^{\infty}(\Omega)} \leq C N^{-(k+1)} \\
\|E-\mathscr{I} E\|_{L^{\infty}\left(I_{j}\right)} & \leq C h_{j}^{k+1}|E|_{k+1, \infty, I_{j}} \\
& \leq C h_{j}^{k+1} \cdot \varepsilon^{-(k+1)} \exp \left(-\frac{\beta x_{j-1}}{\varepsilon}\right) \\
& \leq C\left(N^{-1} \max \left|\psi^{\prime}\right|\right)^{k+1}
\end{aligned}
$$


for any $I_{j}=\left[x_{j-1}, x_{j}\right] \subset \Omega_{1}$. Hence, we obtain

$$
\|E-\mathscr{I} E\|_{L^{\infty}\left(\Omega_{1}\right)} \leq C\left(N^{-1} \max \left|\psi^{\prime}\right|\right)^{k+1} .
$$

From (33), we get

$$
\begin{aligned}
\|E\|_{L^{\infty}\left(\Omega_{2}\right)} & \leq C \max _{x \in[\lambda, 1]} \exp \left(-\frac{\beta x}{\varepsilon}\right) \\
& =C \exp \left(-\frac{\beta \lambda}{\varepsilon}\right) \leq C N^{-(k+1.5)} .
\end{aligned}
$$

Consider the element $K=\left[x_{j-1}, x_{j}\right] \subset \Omega_{2}$. The local nodal functional can be estimated by

$$
\left|N_{i}(E)\right| \leq C \exp \left(-\frac{\beta x_{j-1}}{\varepsilon}\right) ;
$$

thus, we have from the local representation,

$$
\left.\mathscr{I} E\right|_{K}=\sum_{i=0}^{k} N_{i}(E) \varphi_{i}
$$

the estimate

$$
\begin{aligned}
\|\mathscr{J} E\|_{L^{\infty}(K)} & \leq \sum_{i=0}^{k}\left|N_{i}(E)\right| \cdot\left\|\varphi_{i}\right\|_{L^{\infty}(K)} \leq C \exp \left(-\frac{\beta x_{j-1}}{\varepsilon}\right) \\
& \leq C \exp \left(-\frac{\beta \lambda}{\varepsilon}\right) \leq C N^{-(k+1.5)},
\end{aligned}
$$

where we used $\left\|\varphi_{i}\right\|_{L^{\infty}(K)} \leq C, \varphi_{i}$ are basis functions on element $K$. And then, we obtain $\|\mathscr{I} E\|_{L^{\infty}\left(\Omega_{2}\right)} \leq C N^{-(k+1.5)}$. Combining this with (46), we get

$$
\begin{aligned}
\|E-\mathscr{I} E\|_{L^{\infty}\left(\Omega_{2}\right)} & \leq\|E\|_{L^{\infty}\left(\Omega_{2}\right)}+\|\mathscr{I} E\|_{L^{\infty}\left(\Omega_{2}\right)} \\
& \leq C N^{-(k+1.5)} .
\end{aligned}
$$

Collecting (43), (45), and (50), we conclude

$$
\begin{aligned}
\|u-\mathscr{I} u\|_{L^{\infty}\left(\Omega_{i}\right)} & \leq\|S-\mathscr{I S}\|_{L^{\infty}\left(\Omega_{i}\right)}+\|E-\mathscr{I} E\|_{L^{\infty}\left(\Omega_{i}\right)} \\
& \leq\|S-\mathscr{I} S\|_{L^{\infty}(\Omega)}+\|E-\mathscr{I} E\|_{L^{\infty}\left(\Omega_{i}\right)} \\
& \leq \begin{cases}C\left(N^{-1} \max \left|\psi^{\prime}\right|\right)^{k+1}, & \text { if } i=1, \\
C N^{-(k+1)}, & \text { if } i=2 .\end{cases}
\end{aligned}
$$

(ii) The proof of (39): by (38), we easily get

$$
\begin{aligned}
\|u-\mathscr{I} u\|_{L^{2}(\Omega)} & \leq\|u-\mathscr{I} u\|_{L^{\infty}(\Omega)} \cdot|\Omega|^{1 / 2} \\
& \leq C\left(N^{-1} \max \left|\psi^{\prime}\right|\right)^{k+1} .
\end{aligned}
$$

(iii) The proof of (40): by Lemma 1, Lemma 7, and (33), we easily obtain

$$
|S-\mathscr{I S}|_{1, \Omega} \leq C N^{-k}
$$

(iv) The proof of (41): let $x_{j-1 / 2}=\left(x_{j}+x_{j-1}\right) / 2$. Then on the fine part of the mesh, we have

$$
\begin{aligned}
\mid E- & \left.E_{I}\right|_{1, I_{j}} ^{2} \\
\leq & C h_{j}^{2 k}|E|_{k+1, I_{j}}^{2} \\
\leq & C h_{j}^{2 k} \varepsilon^{-(2 k+1)}\left(\exp \left(-\frac{2 \beta x_{j-1}}{\varepsilon}\right)-\exp \left(-\frac{2 \beta x_{j}}{\varepsilon}\right)\right) \\
\leq & C \varepsilon^{-1}\left(N^{-1} \max \left|\psi^{\prime}\right|\right)^{2 k} \exp \left(\frac{2 k \beta x_{j}}{(k+1.5) \varepsilon}\right) \\
& \times \sinh \left(\frac{\beta h_{j}}{\varepsilon}\right) \exp \left(-\frac{2 \beta x_{j-1 / 2}}{\varepsilon}\right),
\end{aligned}
$$

where we have used Lemma 7 and (7). From (9) we get $h_{j} / \varepsilon \leq$ $C$, and therefore $\sinh \left(\beta h_{j} / \varepsilon\right) \leq C \beta h_{j} / \varepsilon$ for $j=1, \ldots, N / 2$. The representation

$$
\frac{\beta h_{j}}{\varepsilon}=\alpha \int_{t_{j-1}}^{t_{j}} \phi^{\prime}(t) \mathrm{d} t \quad \text { for } j=1, \ldots, \frac{N}{2}
$$

yields

$$
\begin{aligned}
\mid E- & \left.E_{I}\right|_{1, I_{j}} ^{2} \\
\leq & C \varepsilon^{-1}\left(N^{-1} \max \left|\psi^{\prime}\right|\right)^{2 k} \exp \left(\frac{2 k \beta x_{j}}{(k+1.5) \varepsilon}\right) \\
& \times \int_{t_{j-1}}^{t_{j}} \frac{-\psi^{\prime}(t)}{\psi(t)} \mathrm{d} t \exp \left(-\frac{2 \beta x_{j-1 / 2}}{\varepsilon}\right) \\
\leq & C \varepsilon^{-1}\left(N^{-1} \max \left|\psi^{\prime}\right|\right)^{2 k} \exp \left(\frac{2 k \beta x_{j}}{(k+1.5) \varepsilon}\right) \frac{1}{\psi\left(t_{j}\right)} \\
& \times \int_{t_{j-1}}^{t_{j}}-\psi^{\prime}(t) \mathrm{d} t \exp \left(-\frac{2 \beta x_{j-1 / 2}}{\varepsilon}\right) \\
\leq & C \varepsilon^{-1}\left(N^{-1} \max \left|\psi^{\prime}\right|\right)^{2 k} \int_{t_{j-1}}^{t_{j}}-\psi^{\prime}(t) \mathrm{d} t \exp \left(\frac{\beta h_{j}}{\varepsilon}\right) \\
\leq & C \varepsilon^{-1}\left(N^{-1} \max \left|\psi^{\prime}\right|\right)^{2 k} \int_{t_{j-1}}^{t_{j}}-\psi^{\prime}(t) \mathrm{d} t,
\end{aligned}
$$

since $\psi>0, \psi^{\prime} \leq 0$ and $\exp \left(\beta h_{j} / \varepsilon\right) \leq C$ by $(9)$. We sum the overall subintervals in the layer region to get

$$
\begin{aligned}
\left|E-E_{I}\right|_{1, \Omega_{1}}^{2} & \leq C \varepsilon^{-1}\left(N^{-1} \max \left|\psi^{\prime}\right|\right)^{2 k}\left(\psi(0)-\psi\left(\frac{1}{2}\right)\right) \\
& \leq C \varepsilon^{-1}\left(N^{-1} \max \left|\psi^{\prime}\right|\right)^{2 k},
\end{aligned}
$$

where $\psi(0)=1$ and $\psi(1 / 2)=1 / N$ are used.

(v) The proof of (42): by triangle inequality

$$
|E-\mathscr{I} E|_{1, \Omega_{2}} \leq|E|_{1, \Omega_{2}}+|\mathscr{I} E|_{1, \Omega_{2}},
$$


we estimate $|E|_{1, \Omega_{2}}$ and $|\mathscr{I} E|_{1, \Omega_{2}}$, respectively. From (33), we obtain

$$
\begin{aligned}
|E|_{1, \Omega_{2}} & \leq C\left(\int_{\lambda}^{1} \varepsilon^{-2} \exp \left(-\frac{2 \beta x}{\varepsilon}\right) \mathrm{d} x\right)^{1 / 2} \\
& \leq C \varepsilon^{-1 / 2} \exp \left(-\frac{\beta \lambda}{\varepsilon}\right) \\
& \leq C \varepsilon^{-1 / 2} N^{-(k+1.5)}
\end{aligned}
$$

An inverse inequality yields

$$
|\mathscr{I} E|_{1, \Omega_{2}} \leq C N\|\mathscr{I} E\|_{L^{2}\left(\Omega_{2}\right)},
$$

and it remains to bound $\|\mathscr{I} E\|_{L^{2}\left(\Omega_{2}\right)}$. Consider the element $K=\left[x_{j-1}, x_{j}\right] \subset \Omega_{2}$. From local representation of $\left.\mathscr{I} E\right|_{K}$, it follows

$$
\begin{aligned}
\|\mathscr{I} E\|_{L^{2}(K)} & \leq \sum_{i=0}^{k}\left|N_{i}(E)\right| \cdot\left\|\varphi_{i}\right\|_{L^{2}(K)} \\
& \leq C N^{-1 / 2} \exp \left(-\frac{\beta x_{j-1}}{\varepsilon}\right),
\end{aligned}
$$

where we used $\left\|\varphi_{i}\right\|_{L^{2}(K)} \leq C N^{-1 / 2}\left\|\widehat{\varphi}_{i}\right\|_{L^{2}(\widehat{K})} \leq C N^{-1 / 2}$. Here $\widehat{K}$ is the reference element of $K$ and $\widehat{\varphi}_{i}$ are the basis functions on $\widehat{K}$. Summing up we get

$$
\begin{aligned}
\|\mathscr{I} E\|_{L^{2}\left(\Omega_{2}\right)}^{2} & =\sum_{K \subset \Omega_{2}}\|\mathscr{I} E\|_{L^{2}(K)}^{2} \\
& \leq C N^{-1} \sum_{j=N / 2+1}^{N} \exp \left(-\frac{2 \beta x_{j-1}}{\varepsilon}\right) .
\end{aligned}
$$

Recall that the mesh size on the coarse mesh has been denoted by $H$ and satisfies $1 / N \leq H \leq 2 / N$. Integrating the inequality

$$
\begin{aligned}
& \exp \left(-\frac{2 \beta x_{j-1}}{\varepsilon}\right)=\exp \left(\frac{2 \beta H}{\varepsilon}\right) \exp \left(-\frac{2 \beta x_{j}}{\varepsilon}\right) \\
& \leq \exp \left(\frac{2 \beta H}{\varepsilon}\right) \exp \left(-\frac{2 \beta x}{\varepsilon}\right) \\
& \text { for } x \in\left[x_{j-1}, x_{j}\right]
\end{aligned}
$$

over $\left(x_{j-1}, x_{j}\right)$ and summing up for $j=N / 2+2, \ldots, N$, we obtain

$$
\begin{gathered}
N^{-1} \exp \left(-\frac{2 \beta x_{j-1}}{\varepsilon}\right) \leq \exp \left(\frac{2 \beta H}{\varepsilon}\right) \int_{x_{j-1}}^{x_{j}} \exp \left(-\frac{2 \beta x}{\varepsilon}\right) \mathrm{d} x, \\
N^{-1} \sum_{j=N / 2+2}^{N} \exp \left(-\frac{2 \beta x_{j-1}}{\varepsilon}\right) \\
\quad \leq \exp \left(\frac{2 \beta H}{\varepsilon}\right) \int_{x_{N / 2+1}}^{1} \exp \left(-\frac{2 \beta x}{\varepsilon}\right) \mathrm{d} x \\
\quad \leq \varepsilon \exp \left(\frac{2 \beta H}{\varepsilon}\right) \exp \left(-\frac{2 \beta x_{N / 2+1}}{\varepsilon}\right) \mathrm{d} x \\
\leq C \varepsilon N^{-2(k+1.5)} .
\end{gathered}
$$

Therefore, we get

$$
\begin{aligned}
\|\mathscr{I} E\|_{L^{2}\left(\Omega_{2}\right)}^{2} & \leq C N^{-1} \exp \left(-\frac{2 \beta x_{N / 2}}{\varepsilon}\right)+C \varepsilon N^{-2(k+1.5)} \\
& \leq C\left(N^{-1}+\varepsilon\right) N^{-2(k+1.5)}
\end{aligned}
$$

And then, we have

$$
|\mathscr{I} E|_{1, \Omega_{2}} \leq C N\left(N^{-1}+\varepsilon\right)^{1 / 2} N^{-(k+1.5)} \leq C N^{-(k+1)} .
$$

Combining this with (59), we conclude (42).

The following statement is the direct consequence of Lemma 8.

Theorem 9. Under the conditions of Lemma 8, one has $\eta=$ $u-\mathscr{I} u$ satisfies

$$
|\|\eta\||_{\varepsilon} \leq C\left(N^{-1} \max \left|\psi^{\prime}\right|\right)^{k}
$$

for S-type mesh.

Proof. Since $u-\mathscr{I} u$ is continuous in $\Omega$, we have $|\eta|_{*}=0$ and $|\eta|_{c}=0$. Then, $\left.\left|\|\eta\|_{\varepsilon}^{2}=\|\eta\|_{0, \Omega}^{2}+\varepsilon\right| \eta\right|_{1, N} ^{2}$. From (40), (41), and (42) of Lemma 8, we obtain

$$
\begin{aligned}
& \varepsilon^{1 / 2}|u-\mathscr{I} u|_{1, \Omega} \\
& \quad \leq \varepsilon^{1 / 2}\left(|S-\mathscr{I S}|_{1, \Omega}+|E-\mathscr{I} E|_{1, \Omega_{1}}+|E-\mathscr{I} E|_{1, \Omega_{2}}\right) \\
& \quad \leq C\left(N^{-1} \max \left|\psi^{\prime}\right|\right)^{k} .
\end{aligned}
$$

Using this together with (39), we easily conclude the result of Theorem 9.

Now we turn to estimate $|\|\xi\||_{\varepsilon}$.

Lemma 10. Under the conditions of Lemma 8, one has

$$
\begin{gathered}
\left|\int_{\Omega} b(S-\mathscr{I} S) \xi^{\prime} \mathrm{d} x\right| \leq C N^{-(k+1 / 2)} \mid\|\xi\|_{\varepsilon^{\prime}} \\
\left|\int_{\Omega} b(E-\mathscr{I} E) \xi^{\prime} \mathrm{d} x\right| \leq C C_{\psi}\left(N^{-1} \max \left|\psi^{\prime}\right|\right)^{k+1 / 2} \mid\|\xi\|_{\mathcal{E}},
\end{gathered}
$$

where $C_{\psi}:=1+\left(N^{-1} \max \left|\psi^{\prime}\right| \ln N\right)^{1 / 2}$.

Proof. (i) The proof of (69): by Lemma 8 and integrating by parts, we get

$$
\begin{aligned}
& \left|\int_{\Omega} b(S-\mathscr{I S}) \xi^{\prime} \mathrm{d} x\right| \\
& \quad \leq\|S-\mathscr{I} S\|_{L^{\infty}(\Omega)}\left|\sum_{j=1}^{N} \int_{I_{j}} b \xi^{\prime} \mathrm{d} x\right| \\
& \quad \leq C N^{-(k+1)}\left|\sum_{j=1}^{N}\left[(b \xi)\left(x_{j}^{-}\right)-(b \xi)\left(x_{j-1}^{+}\right)\right]-\int_{\Omega} b^{\prime} \xi \mathrm{d} x\right|
\end{aligned}
$$




$$
\begin{aligned}
\leq & C N^{-(k+1)}\left|\sum_{j=1}^{N}\left[(b \xi)\left(x_{j}^{-}\right)-(b \xi)\left(x_{j-1}^{+}\right)\right]\right| \\
& +C N^{-(k+1)}\left\|b^{\prime}\right\|_{L^{2}(\Omega)}\|\xi\|_{L^{2}(\Omega)} .
\end{aligned}
$$

Recalling that $\xi=\mathscr{I} u-U_{N}$ is continuous in $\Omega_{1}$, we have $\left[\xi\left(x_{j}\right)\right]=0, j=0, \ldots, N / 2-1$. And then,

$$
\begin{aligned}
\mid \sum_{j=1}^{N}[ & \left.(b \xi)\left(x_{j}^{-}\right)-(b \xi)\left(x_{j-1}^{+}\right)\right] \mid \\
\leq & b\left(x_{0}\right)\left|\xi\left(x_{0}^{+}\right)\right| \\
& +\sum_{j=1}^{N-1} b\left(x_{j}\right)\left|\left[\xi\left(x_{j}\right)\right]\right|+b\left(x_{N}^{-}\right)\left|\xi\left(x_{N}^{-}\right)\right| \\
= & \sum_{j=N / 2}^{N-1} b\left(x_{j}\right)\left|\left[\xi\left(x_{j}\right)\right]\right|+b\left(x_{N}^{-}\right)\left|\xi\left(x_{N}^{-}\right)\right| \\
\leq & \left(\sum_{j=N / 2}^{N} b\left(x_{j}\right)\right)^{1 / 2} \\
& \times\left(\sum_{j=N / 2}^{N-1} b\left(x_{j}\right)\left[\xi\left(x_{j}\right)\right]^{2}+b\left(x_{N}^{-}\right) \xi\left(x_{N}^{-}\right)^{2}\right)^{1 / 2} \\
\leq & C N^{1 / 2}|\xi|_{c} \cdot
\end{aligned}
$$

Combining this with (71), we can easily get (69).

(ii) The proof of (70): by Cauchy-Schwarz inequality,

$$
\begin{aligned}
\left|\int_{\Omega} b(E-\mathscr{I} E) \xi^{\prime} \mathrm{d} x\right| \leq & C\|E-\mathscr{I} E\|_{L^{2}\left(\Omega_{1}\right)}|\xi|_{1, \Omega_{1}} \\
& +C\|E-\mathscr{I} E\|_{L^{2}\left(\Omega_{2}\right)}|\xi|_{1, \Omega_{2}},
\end{aligned}
$$

where by Lemma 7 ,

$$
\begin{aligned}
\|E-\mathscr{I} E\|_{L^{2}\left(\Omega_{1}\right)} & \leq\left|\Omega_{1}\right|^{1 / 2}\|E-\mathscr{I} E\|_{L^{\infty}\left(\Omega_{1}\right)} \\
& \leq C(\varepsilon \ln N)^{1 / 2}\left(N^{-1} \max \left|\psi^{\prime}\right|\right)^{k+1},
\end{aligned}
$$

and from (33), (65),

$$
\begin{aligned}
\|E-\mathscr{I} E\|_{L^{2}\left(\Omega_{2}\right)} \leq & \|E\|_{L^{2}\left(\Omega_{2}\right)}+\|\mathscr{I} E\|_{L^{2}\left(\Omega_{2}\right)} \\
\leq & C \varepsilon^{1 / 2} N^{-(k+1)} \\
& +C\left(N^{-1 / 2}+\varepsilon^{1 / 2}\right) N^{-(k+1.5)} \\
\leq & C\left(\varepsilon^{1 / 2} N^{-(k+1)}+N^{-(k+2)}\right) .
\end{aligned}
$$

Thus, we obtain

$$
\begin{aligned}
& \left|\int_{\Omega} b(E-\mathscr{I} E) \xi^{\prime} \mathrm{d} x\right| \\
& \quad \leq C \varepsilon^{1 / 2}\left[(\ln N)^{1 / 2}\left(N^{-1} \max \left|\psi^{\prime}\right|\right)^{k+1}+N^{-(k+1)}\right]|\xi|_{1, \Omega} \\
& \quad+C N^{-(k+2)}|\xi|_{1, \Omega_{2}}
\end{aligned}
$$

$$
\begin{aligned}
\leq & C\left[(\ln N)^{1 / 2}\left(N^{-1} \max \left|\psi^{\prime}\right|\right)^{k+1}+N^{-(k+1)}\right] \\
& \times \mid\|\xi\|_{\varepsilon}+C N^{-(k+2)} \cdot N\|\xi\|_{L^{2}\left(\Omega_{2}\right)} \\
\leq & \left.C\left[(\ln N)^{1 / 2}\left(N^{-1} \max \left|\psi^{\prime}\right|\right)^{k+1}+N^{-(k+1)}\right]\|\xi\|\right|_{\varepsilon} \\
\leq & C C_{\psi}\left(N^{-1} \max \left|\psi^{\prime}\right|\right)^{k+1 / 2} \mid\|\xi\|_{\varepsilon^{\prime}}
\end{aligned}
$$

where $C_{\psi}:=1+\left(N^{-1} \max \left|\psi^{\prime}\right| \ln N\right)^{1 / 2}$.

Remark 11. The factor $C_{\psi}$ is bounded by a constant for all meshes listed in Table 1. But there also exists counterexample that $C_{\psi}$ increases with the increasing of $N$; see [21] for details.

Theorem 12. Under the conditions of Lemma 8. Assuming $\alpha=$ $\mathcal{O}(1 / H)$, then $\xi=\mathscr{I} u-U_{N}$ satisfies

$$
\mid\|\xi\|_{\varepsilon} \leq C C_{\psi}\left(N^{-1} \max \left|\psi^{\prime}\right|\right)^{k+1 / 2} .
$$

Proof. By Lemma 3 and Lemma 4, we first obtain

$$
\begin{aligned}
C_{1} \mid\|\xi\|_{\varepsilon}^{2} & \leq \mathscr{A}_{N}(\xi, \xi)=-\mathscr{A}_{N}(\eta, \xi) \\
& =-\mathscr{B}_{N}(\eta, \xi)-\mathscr{C}_{N}(\eta, \xi)-\mathcal{S}_{N}(\eta, \xi) .
\end{aligned}
$$

By the definition of $\mathscr{I} u$, we have $\eta\left(x_{j}\right)=0, j=0,1, \ldots, N$. Consequently,

$$
\begin{aligned}
\mathscr{B}_{N}(\eta, \xi)= & \int_{\Omega} \varepsilon \eta^{\prime}\left(\xi^{\prime}+\mathscr{L}_{1}(\xi)\right) \mathrm{d} x, \\
\mathscr{C}_{N}(\eta, \xi)= & \int_{\Omega} b \eta \xi^{\prime} \mathrm{d} x \\
& +\int_{\Omega}\left(c+b^{\prime}\right) \eta \xi \mathrm{d} x \equiv I_{1}+I_{2}, \\
& \mathcal{S}_{N}(\eta, \xi)=0 .
\end{aligned}
$$

Firstly, we consider the term $\mathscr{B}_{N}(\eta, \xi)$. By Lemma 7 , Lemma 8, and (29), we have

$$
\begin{aligned}
\left|\mathscr{B}_{N}(\eta, \xi)\right| & =\left|\int_{\Omega_{2}} \varepsilon \eta^{\prime} \mathscr{L}_{1}(\xi) \mathrm{d} x\right| \leq C \varepsilon\left\|\eta^{\prime}\right\|_{L^{2}\left(\Omega_{2}\right)}|\xi|_{*} \\
& \leq C \varepsilon^{1 / 2}\left(|S-\mathscr{I S}|_{1, \Omega_{2}}+|E-\mathscr{I} E|_{1, \Omega_{2}}\right) \mid\|\xi\|_{\varepsilon} \\
& \leq C \varepsilon^{1 / 2} N^{-k} \mid\|\xi\|_{\varepsilon^{*}}
\end{aligned}
$$

Now consider the term $\mathscr{C}_{N}(\eta, \xi)$. By Lemma 10 , we get

$$
\begin{aligned}
\left|I_{1}\right| & \leq\left|\int_{\Omega} b(S-\mathscr{I} S) \xi^{\prime} \mathrm{d} x\right|+\left|\int_{\Omega} b(E-\mathscr{I} E) \xi^{\prime} \mathrm{d} x\right| \\
& \leq C\left[N^{-(k+1 / 2)}+C_{\psi}\left(N^{-1} \max \left|\psi^{\prime}\right|\right)^{k+1 / 2}\right] \mid\|\xi\|_{\varepsilon} \\
& \leq C C_{\psi}\left(N^{-1} \max \left|\psi^{\prime}\right|\right)^{k+1 / 2} \mid\|\xi\|_{\varepsilon^{\prime}} .
\end{aligned}
$$

By (39), the second term in the right hand side of (79) can be easily estimated with

$$
\left|I_{2}\right| \leq C\|\eta\|_{0, \Omega}\|\xi\|_{0, \Omega} \leq C\left(N^{-1} \max \left|\psi^{\prime}\right|\right)^{k+1} \mid\|\xi\|_{\varepsilon} .
$$


TABLE 1: Some examples of mesh-generating and mesh-characterizing functions of S-type meshes.

\begin{tabular}{|c|c|c|c|c|}
\hline Name & $\phi(t)$ & $\max \phi^{\prime}$ & $\psi(t)$ & $\max \left|\psi^{\prime}\right|$ \\
\hline S-mesh & $2 t \ln N$ & $2 \ln N$ & $N^{-2 t}$ & $2 \ln N$ \\
\hline B-S mesh & $-\ln (1-2 t(1-1 / N))$ & $2 N$ & $1-2 t(1-1 / N)$ & 2 \\
\hline Polynomial S-mesh & $(2 t)^{m} \ln N$ & $2 m \ln N$ & $N^{-(2 t)^{m}}$ & $C(\ln N)^{1 / m}$ \\
\hline Modified B-S mesh & $t /(q-t), q=1 / 2(1+1 / \ln N)$ & $3 \ln ^{2} N$ & $e^{-t /(q-t)}$ & $3 /(2 q) \leq 3$ \\
\hline
\end{tabular}

TABLE 2: History of convergence of the FEM/LDG coupled method, $\varepsilon=10^{-6}$.

\begin{tabular}{|c|c|c|c|c|c|c|c|c|c|}
\hline \multirow{3}{*}{$k$} & \multirow{3}{*}{$N$} & \multicolumn{4}{|c|}{\|\|$u-u_{N} \|\left.\right|_{\varepsilon}$} & \multicolumn{4}{|c|}{\|\|$u_{I}-u_{N} \|\left.\right|_{\varepsilon}$} \\
\hline & & \multicolumn{2}{|c|}{ S-mesh } & \multicolumn{2}{|c|}{ B-S mesh } & \multicolumn{2}{|c|}{ S-mesh } & \multicolumn{2}{|c|}{ B-S mesh } \\
\hline & & Error & ln-ord & Error & ord & Error & ln-ord & Error & ord \\
\hline \multirow{6}{*}{1} & 16 & $2.060 e-01$ & - & $9.204 e-02$ & - & $3.539 e-02$ & - & $6.912 e-03$ & - \\
\hline & 32 & $1.304 e-01$ & 0.97 & $4.750 e-02$ & 0.95 & $1.429 e-02$ & 1.93 & $2.069 e-03$ & 1.74 \\
\hline & 64 & $7.868 e-02$ & 0.99 & $2.412 e-02$ & 0.98 & $5.217 e-03$ & 1.97 & $6.378 e-04$ & 1.70 \\
\hline & 128 & $4.599 e-02$ & 1.00 & $1.216 e-02$ & 0.99 & $1.785 e-03$ & 1.99 & $2.051 e-04$ & 1.64 \\
\hline & 256 & $2.630 e-02$ & 1.00 & $6.101 e-03$ & 1.00 & $5.846 e-04$ & 2.00 & $6.845 e-05$ & 1.58 \\
\hline & 512 & $1.480 e-02$ & 1.00 & $3.057 e-03$ & 1.00 & $1.854 e-04$ & 2.00 & $2.344 e-05$ & 1.55 \\
\hline \multirow{6}{*}{2} & 16 & $4.230 e-02$ & - & $8.054 e-03$ & - & $6.413 e-03$ & - & $7.305 e-04$ & - \\
\hline & 32 & $1.740 e-02$ & 1.89 & $2.151 e-03$ & 1.90 & $1.681 e-03$ & 2.85 & $1.022 e-04$ & 2.84 \\
\hline & 64 & $6.406 e-03$ & 1.96 & $5.553 e-04$ & 1.95 & $3.744 e-04$ & 2.94 & $1.360 e-05$ & 2.91 \\
\hline & 128 & $2.198 e-03$ & 1.98 & $1.410 e-04$ & 1.98 & $7.518 e-05$ & 2.98 & $1.777 e-06$ & 2.94 \\
\hline & 256 & $7.199 e-04$ & 1.99 & $3.554 e-05$ & 1.99 & $1.409 e-05$ & 2.99 & $2.325 e-07$ & 2.93 \\
\hline & 512 & $2.280 e-04$ & 2.00 & $8.919 e-06$ & 1.99 & $2.510 e-06$ & 3.00 & $3.267 e-08$ & 2.83 \\
\hline
\end{tabular}

This, combined with (81), yields

$$
\left|\mathscr{C}_{N}(\eta, \xi)\right| \leq C C_{\psi}\left(N^{-1} \max \left|\psi^{\prime}\right|\right)^{k+1 / 2} \mid\|\xi\|_{\varepsilon} .
$$

Collecting (78), (80), and (83), we conclude Theorem 12.

Remark 13. Uniform convergence of higher order LDG method on 2D Shishkin-mesh was considered in [15]. From Theorem 3.1 of [15], a similar result can be obtained as our Theorem 12. From Theorem 3.1 and Remark 3.3 of [15], we can find the following conditions must hold: $0 \leq C_{11} \leq \mathscr{O}(1)$ on $\mathscr{E}$ or $0 \leq C_{11} \leq \mathscr{O}(N)$ on $\mathscr{E}_{+}^{B}$ and $C_{11}=0$ on $\mathscr{E} \backslash \mathscr{E}_{+}^{B}$. Here $C_{11}$ is a parameter in the definition of numerical fluxes, and $\mathscr{E}, \mathscr{E}_{+}^{B}$ are unions of some edges of elements. In our paper, a parameter $\alpha$ in (18), which plays the same role as $C_{11}$, takes value as $\alpha=\mathcal{O}(1 / H)$. This does not fulfill the condition of Theorem 3.1 of [15].

The combination of Theorem 9 and Theorem 12 leads to our main results directly, that is, the following.

Theorem 14. Let $u$ and $U_{N}$ be the solutions of the continuous problem (10) and the discrete problem (25), respectively. Assume that the piecewise differential mesh-generating function $\phi$ satisfies (6). Taking $\alpha=\mathcal{O}(1 / H)$, then

$$
\left.\left\|u-U_{N}\right\|\right|_{\varepsilon} \leq C C_{\psi}\left(N^{-1} \max \left|\psi^{\prime}\right|\right)^{k} .
$$

Corollary 15. Let $\left(Q_{N}, U_{N}\right)$ be the solution obtained by the coupled method (13)-(15) with numerical fluxes (17)-(19). Under the assumption of Theorem 14, one has

$$
\left|q-Q_{N}, u-U_{N}\right|_{\mathscr{A}_{N}} \leq C C_{\psi}\left(N^{-1} \max \left|\psi^{\prime}\right|\right)^{k},
$$

where $|(\cdot, \cdot)|_{\mathscr{A}_{N}}$ is a problem-related norm defined by

$$
|(r, v)|_{\mathscr{A}_{N}}^{2}=\|v\|_{0, \Omega}^{2}+\varepsilon\|r\|_{0, \Omega_{2}}^{2}+\varepsilon\left\|\left(v^{1}\right)^{\prime}\right\|_{0, \Omega_{1}}^{2}+\varepsilon|v|_{*}^{2}+|v|_{c}^{2} .
$$

Proof. From (23), we have $q-Q_{N}=\left(u^{2}-U_{N}^{2}\right)^{\prime}-\mathscr{L}_{1}\left(U_{N}\right)$. Since $\mathscr{L}_{1}(u)=0$, we obtain $q-Q_{N}=\left(u^{2}-U_{N}^{2}\right)^{\prime}+\mathscr{L}_{1}(u-$ $\left.U_{N}\right)$. In terms of (29), we conclude $\left|\left(q-Q_{N}, u-U_{N}\right)\right|_{\mathscr{A}_{N}} \leq$ $C\left|\left\|u-U_{N}\right\|\right|_{\varepsilon}$, which implies the conclusion.

\section{Numerical Experiments}

In this section, we numerically verify the sharpness of our theoretical findings. In our numerical experiments, we take $\alpha=1 / H, \beta=1 / 2$ in (17) and (18).

Example 16. We solve the model problem (1) with $b=3-x$, $c=1$, and taking $f$ such that the exact solution is

$$
u(x)=\left(1-e^{-2 x / \varepsilon}\right) \sin (1-x),
$$

which exhibits a boundary layer with the width $\mathcal{O}(\varepsilon \ln (1 / \varepsilon))$ at the outflow boundary $x=0$.

In the following, "ln-ord" denotes the exponent $r$ in a convergence order of the form $\mathcal{O}\left(\left(N^{-1} \ln N\right)^{r}\right)$, while "ord" denotes the exponent $r$ in a convergence order of the form $\mathcal{O}\left(N^{-r}\right)$.

The errors $\left|\left\|u-u_{N}\right\|\right|_{\varepsilon}$ and $\left|\left\|u_{I}-u_{N}\right\|\right|_{\varepsilon}$ for the FEM/LDG coupled method with higher order $k$ th elements are shown in Table 2. We have chosen $\varepsilon=10^{-6}$ in our calculations on 
S-meshes and B-S meshes. From Table 2, we observe that the numerical results for $\left|\left\|u-u_{N}\right\|\right|_{\varepsilon}$ agree with those predicted in Theorem 14. Note that the closeness errors $\left|\left\|u_{I}-u_{N}\right\|\right|_{\varepsilon}$ have a supercloseness property of order $k+1$ if $k$ th polynomial is used. This phenomenon indicates that the supercloseness result of order $k+1 / 2$ proved in Theorem 12 is not optimal.

\section{Conflict of Interests}

The authors declare that there is no conflict of interests regarding the publication of this paper.

\section{Acknowledgments}

This work is supported by Zhejiang Provincial Natural Science Foundation of China (Grant no. LQ12A01014) and Zhejiang Provincial Department of Education (Grant no. Y201330020).

\section{References}

[1] Z. Zhang, "Finite element superconvergence on Shishkin mesh for 2-D convection-diffusion problems," Mathematics of Computation, vol. 72, no. 243, pp. 1147-1177, 2003.

[2] G. I. Shishkin, Grid approximation of singularly perturbed elliptic and parabolic equations [Ph.D. thesis], Keldysh Institue, Moscow, Russia, 1990 (Russian).

[3] T. Linß, "An upwind difference scheme on a novel Shishkintype mesh for a linear convection-diffusion problem," Journal of Computational and Applied Mathematics, vol. 110, no. 1, pp. 93-104, 1999.

[4] T. Linss, "Analysis of a Galerkin finite element method on a Bakhvalov-Shishkin mesh for a linear convection-diffusion problem," IMA Journal of Numerical Analysis, vol. 20, no. 4, pp. 621-632, 2000.

[5] M. Stynes and E. O’Riordan, “A uniformly convergent Galerkin method on a Shishkin mesh for a convection-diffusion problem," Journal of Mathematical Analysis and Applications, vol. 214, no. 1, pp. 36-54, 1997.

[6] H. G. Roos and T. Linß, "Sufficient conditions for uniform convergence on layer-adapted grids," Computing, vol. 63, no. 1, pp. 27-45, 1999.

[7] H. G. Roos and H. Zarin, "A supercloseness result for the discontinuous Galerkin stabilization of convection-diffusion problems on Shishkin meshes," Numerical Methods for Partial Differential Equations, vol. 23, no. 6, pp. 1560-1576, 2007.

[8] Z. Xie and Z. Zhang, "Superconvergence of DG method for one-dimensional singularly perturbed problems," Journal of Computational Mathematics, vol. 25, no. 2, pp. 185-200, 2007.

[9] Z. Xie, Z. Zhang, and Z. Zhang, "A numerical study of uniform superconvergence of LDG method for solving singularly perturbed problems," Journal of Computational Mathematics, vol. 27, no. 2-3, pp. 280-298, 2009.

[10] Z. Xie and Z. Zhang, "Uniform superconvergence analysis of the discontinuous Galerkin method for a singularly perturbed problem in 1-D," Mathematics of Computation, vol. 79, no. 269, pp. 35-45, 2010.

[11] H. Zarin and H. G. Roos, "Interior penalty discontinuous approximations of convection-diffusion problems with parabolic layers," Numerische Mathematik, vol. 100, no. 4, pp. 735-759, 2005.

[12] H. Zarin, "Continuous-discontinuous finite element method for convection-diffusion problems with characteristic layers," Journal of Computational and Applied Mathematics, vol. 231, no. 2, pp. 626-636, 2009.

[13] H. Zhu, H. Tian, and Z. Zhang, "Convergence analysis of the LDG method for singularly perturbed two-point boundary value problems," Communications in Mathematical Sciences, vol. 9, no. 4, pp. 1013-1032, 2011.

[14] H. Zhu and Z. Zhang, "Convergence analysis of the LDG method applied to singularly perturbed problems," Numerical Methods for Partial Differential Equations, vol. 29, no. 2, pp. 396421, 2013.

[15] H. Zhu and Z. Zhang, "Uniform convergence of the LDG method for a singularly perturbed problem with the exponential boundary layer," Mathematics of Computation, vol. 83, no. 286, pp. 635-663, 2014.

[16] D. N. Arnold, F. Brezzi, B. Cockburn, and L. D. Marini, "Unified analysis of discontinuous Galerkin methods for elliptic problems," SIAM Journal on Numerical Analysis, vol. 39, no. 5, pp. 1749-1779, 2001/02.

[17] P. Zhu, Z. Xie, and S. Zhou, "A uniformly convergent continuous-discontinuous Galerkin method for singularly perturbed problems of convection-diffusion type," Applied Mathematics and Computation, vol. 217, no. 9, pp. 4781-4790, 2011.

[18] Z. Q. Xie, P. Zhu, and S. Z. Zhou, "Uniform convergence of a coupled method for convection-diffusion problems in 2-D Shishkin mesh," International Journal of Numerical Analysis and Modeling, vol. 10, no. 4, pp. 845-859, 2013.

[19] P. Zhu and S. Xie, "Higher order uniformly convergent continuous/discontinuous Galerkin methods for singularly perturbed problems of convection-diffusion type," Applied Numerical Mathematics, vol. 76, pp. 48-59, 2014.

[20] S. Franz, "Convergence phenomena of $Q_{p}$-elements for convection-diffusion problems," Numerical Methods for Partial Differential Equations, vol. 29, no. 1, pp. 280-296, 2013.

[21] S. Franz and G. Matthies, "Local projection stabilisation on Stype meshes for convection-diffusion problems with characteristic layers," Computing. Archives for Scientific Computing, vol. 87, no. 3-4, pp. 135-167, 2010.

[22] P. Zhu, Z. Xie, and S. Zhou, "A coupled continuous-discontinuous FEM approach for convection diffusion equations," Acta Mathematica Scientia B, vol. 31, no. 2, pp. 601-612, 2011.

[23] P. Castillo, B. Cockburn, I. Perugia, and D. Schötzau, "An a priori error analysis of the local discontinuous Galerkin method for elliptic problems," SIAM Journal on Numerical Analysis, vol. 38, no. 5, pp. 1676-1706, 2000.

[24] B. Cockburn and C. W. Shu, "The local discontinuous Galerkin method for time-dependent convection-diffusion systems," SIAM Journal on Numerical Analysis, vol. 35, no. 6, pp. 24402463, 1998.

[25] I. Perugia and D. Schötzau, "On the coupling of local discontinuous Galerkin and conforming finite element methods," Journal of Scientific Computing, vol. 16, no. 4, pp. 411-433, 2001.

[26] L. Tobiska, "Analysis of a new stabilized higher order finite element method for advection-diffusion equations," Computer Methods in Applied Mechanics and Engineering, vol. 196, no. 13, pp. 538-550, 2006. 
[27] H.-G. Roos, M. Stynes, and L. Tobiska, Robust Numerical Methods for Singularly Perturbed Differential Equations, vol. 24 of Springer Series in Computational Mathematics, Springer, Berlin, Germany, 2nd edition, 2008. 


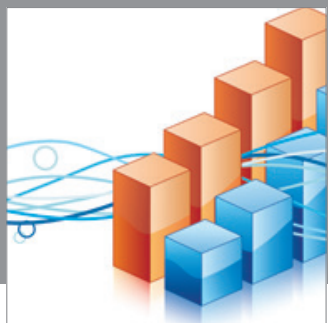

Advances in

Operations Research

mansans

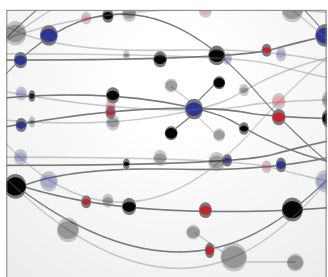

The Scientific World Journal
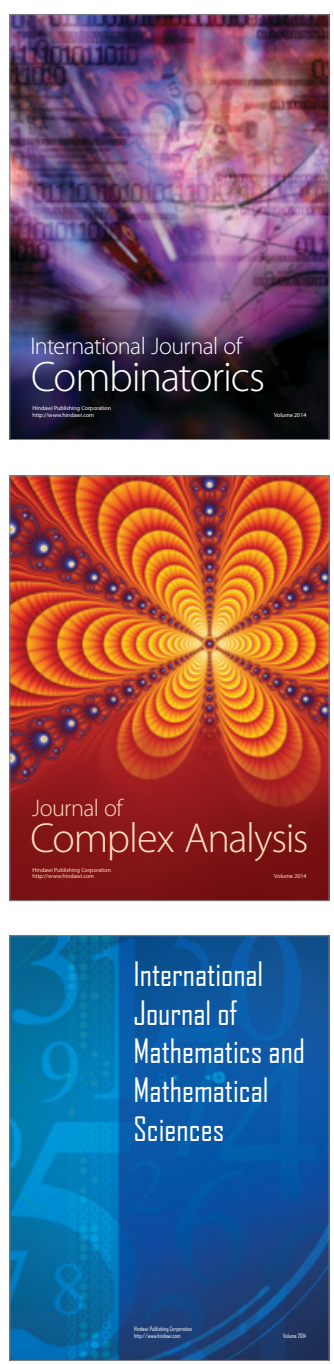
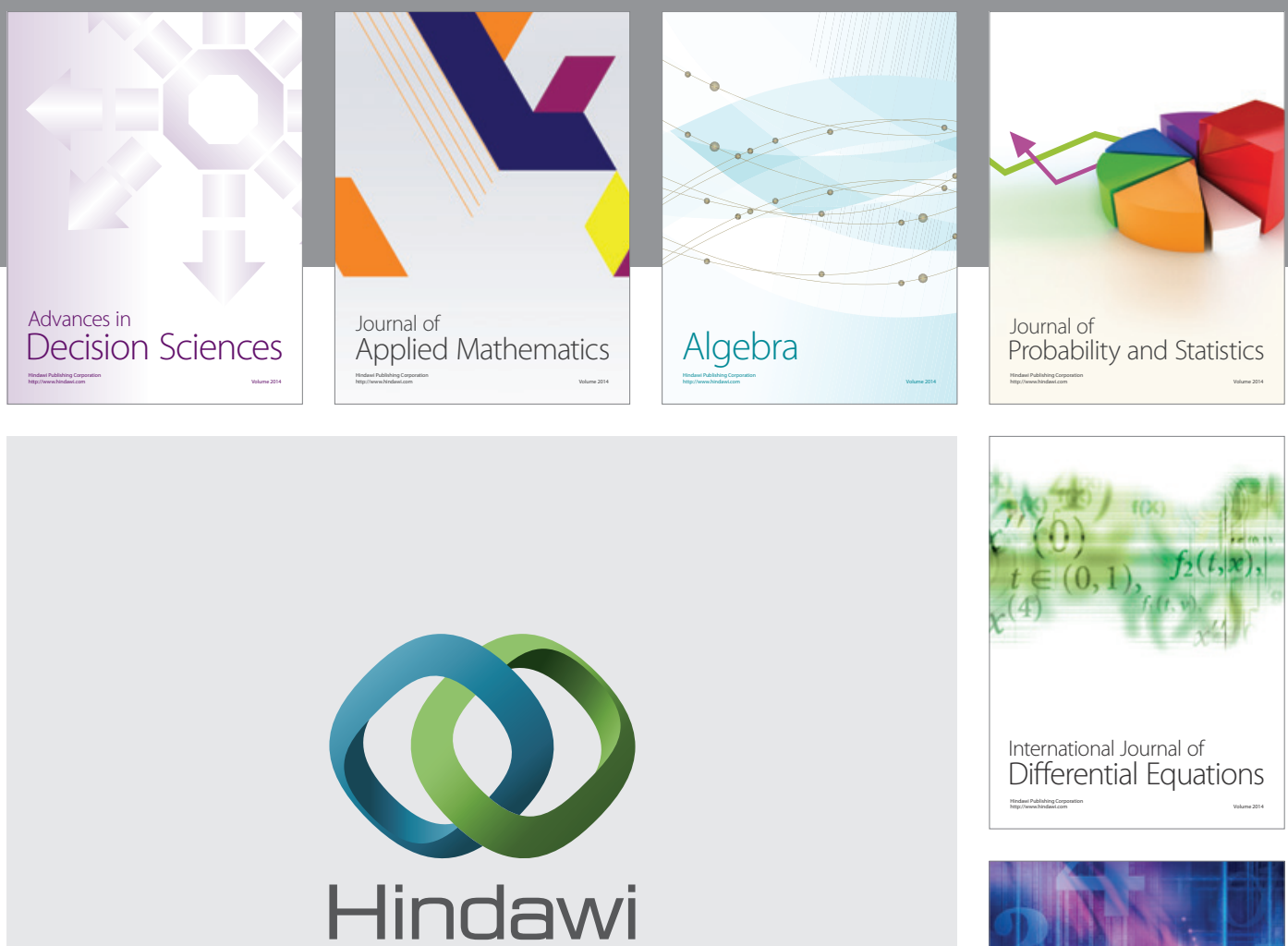

Submit your manuscripts at http://www.hindawi.com
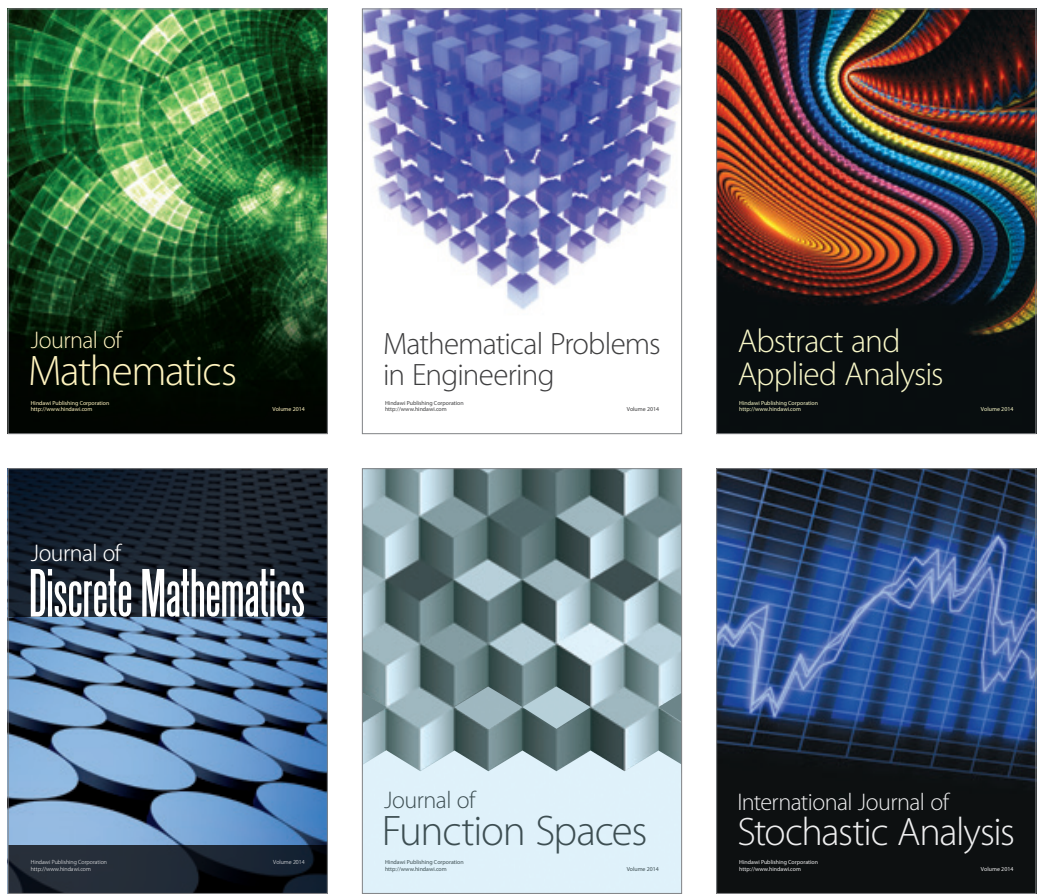

Journal of

Function Spaces

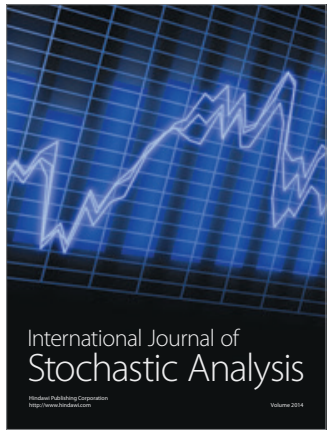

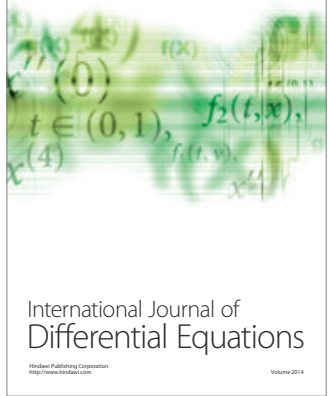
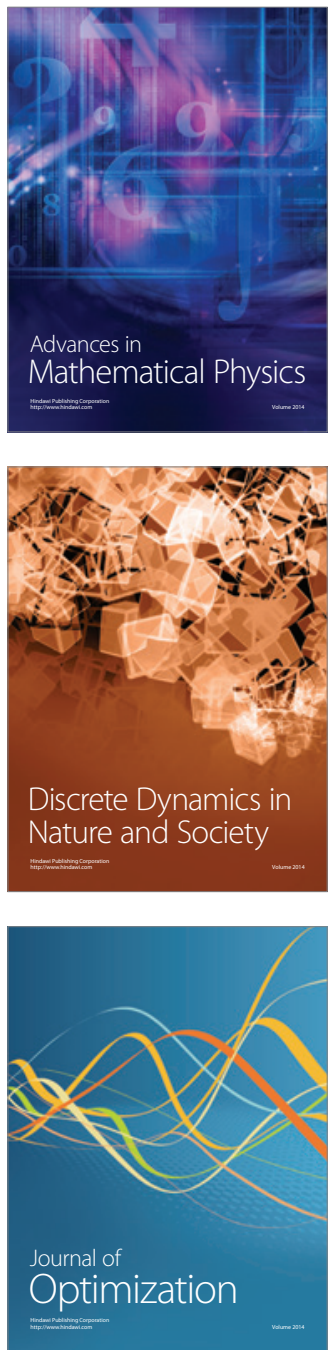